\title{
Management von Blutungen und Infektionen im Rahmen viszeralchirurgischer Operationen
}

Die chirurgische Therapie von Malignomen hat sich zusammen mit der neoadjuvanten Radiochemotherapie in den letzten Jahren stetig weiterentwickelt und ermöglicht im Rahmen multiviszeraler Tumorresektionen eine kurative Krebstherapie [1, 2]. Im Rahmen der Sarkomchirurgie ist die Mitversorgung von Gefäßen bis hin zum Gefäßersatz elementar [3]. Auch die minimalinvasive Chirurgie (MIC) hat sich in vielen Bereichen zur Therapie der 1 . Wahl etabliert [4].

Blutungen und Infektionen von viszeralen Gefäßen bzw. Aorta und unterer Hohlvene im Abdomen und Retroperitoneum stellen in diesem $\mathrm{Zu}$ sammenhang seltene, aber schwerwiegende Komplikationen dar und erfordern neben chirurgischer bzw. gefäßchirurgischer Expertise auch Kenntnisse elementarer Behandlungskonzepte wie z. B. Blutgerinnungsmanagement [5]. Dies wird deutlich, wenn man sich die heute zur Verfügung stehende multimodale Gefäßchirurgie vor Augen führt. Neben etablierten klassischen chirurgischen Operationstechniken stehen dem Operateur bei Blutungen mittlerweile auch interventionelle, endovaskuläre Techniken wie z. B. Coil-Embolisation oder gecoverte Stents (Stentgrafts) zur Verfügung [6]. Selbst das Peritoneum wird als autologer Venenersatz in der Pankreas- und hepatobiliären Chirurgie herangezogen [7].

Die Kombination dieser Techniken wird als Hybridoperation bezeichnet und kann entweder in Kooperation mit interventionellen Radiologen oder eigenständig durchgeführt werden. Voraussetzungen hierfür sind eine entsprechende Ausstattung des Operationssaales mit Durchleuchtungs- und Angiographieeinheit, im Idealfall ein Hybridoperationsraum, des Weiteren aber auch Kenntnisse in Materialkunde und Indikationsstellung zur Verfahrenswahl.

Gefäßinfektionen stellen eine ganz besondere Herausforderung dar, bei der die konventionelle Gefäßchirurgie Therapie der 1. Wahl darstellt und endovaskuläre Techniken als „bridging“, d. h. als überbrückende Maßnahme einzustufen sind, da bez. alloplastischen Gefäßersatzmaterials die alte Devise gilt: „leave nothing behind“. Bisweilen sind zweibzw. mehrzeitige Operationen notwendig und sinnvoll. Vor diesem Hintergrund entscheiden oft die chirurgische Strategie und das übergeordnete Behandlungskonzept über das operative Ergebnis und das Überleben des Patienten.

Die Therapie der aortoenteralen Fistel, mit oder ohne Blutung, stellt in diesem Zusammenhang ein Paradebeispiel für die Notwendigkeit einer interdisziplinären, multimodalen Vorgehensweise dar und wird im Folgenden ebenfalls abgehandelt [8].

Der folgende Artikel fokussiert somit aus gefäßchirurgischer Sicht auf das intraoperative und peri- bzw. postoperative Management von Blutungen und Infektionen, die im Rahmen viszeralchirurgischer/abdomineller Operationen auftreten.

\section{Management intraoperativer Blutungen}

Blutungen entstehen bei Verletzungen 2. Grades und 3. Grades, d.h. penetrierenden und perforierenden Verletzungen der Gefäßwand von außen nach innen (• Tab. 1). Primäres Ziel der Blutstillung und der Reparatur arterieller und venöser Blutungen ist zunächst die Vermeidung des hämorrhagischen Schocks und dessen Folgen (Multiorganversagen, Gerinnungsstörung, Tod). In Anlehnung an die Einteilung der Gefäßverletzungen nach Vollmar (- Abb. 1) handelt sich bei intraoperativen Blutungen in der Regel um direkte Verletzungen mit scharfem Trauma.

\begin{tabular}{|ll|}
\hline Tab. 1 Einteilung der Verletzungsschweregrade von Gefäßen \\
\hline Grad I & $\begin{array}{l}\text { Verletzung der Adventitia, evtl. auch der Media; keine Eröffnung des Gefäßlumens, keine } \\
\text { Blutung oder Ischämie }\end{array}$ \\
\hline Grad II & $\begin{array}{l}\text { Spalt- oder lochförmige Eröffnung des Gefäßlumens; partiell erhaltene Gefäßkontinuität, } \\
\text { Manifestation durch Blutung }\end{array}$ \\
\hline Grad III & $\begin{array}{l}\text { Vollständige Durchtrennung des Gefäßes, bei }<8 \mathrm{~mm} \text { Gefäßdurchmesser evtl. Retraktion } \\
\text { und Intimaeinrollen mit Sistieren der Blutung, }>8 \mathrm{~mm} \text { erheblicher Blutverlust und Ischä- } \\
\text { mien des Endorgans }\end{array}$ \\
\hline
\end{tabular}




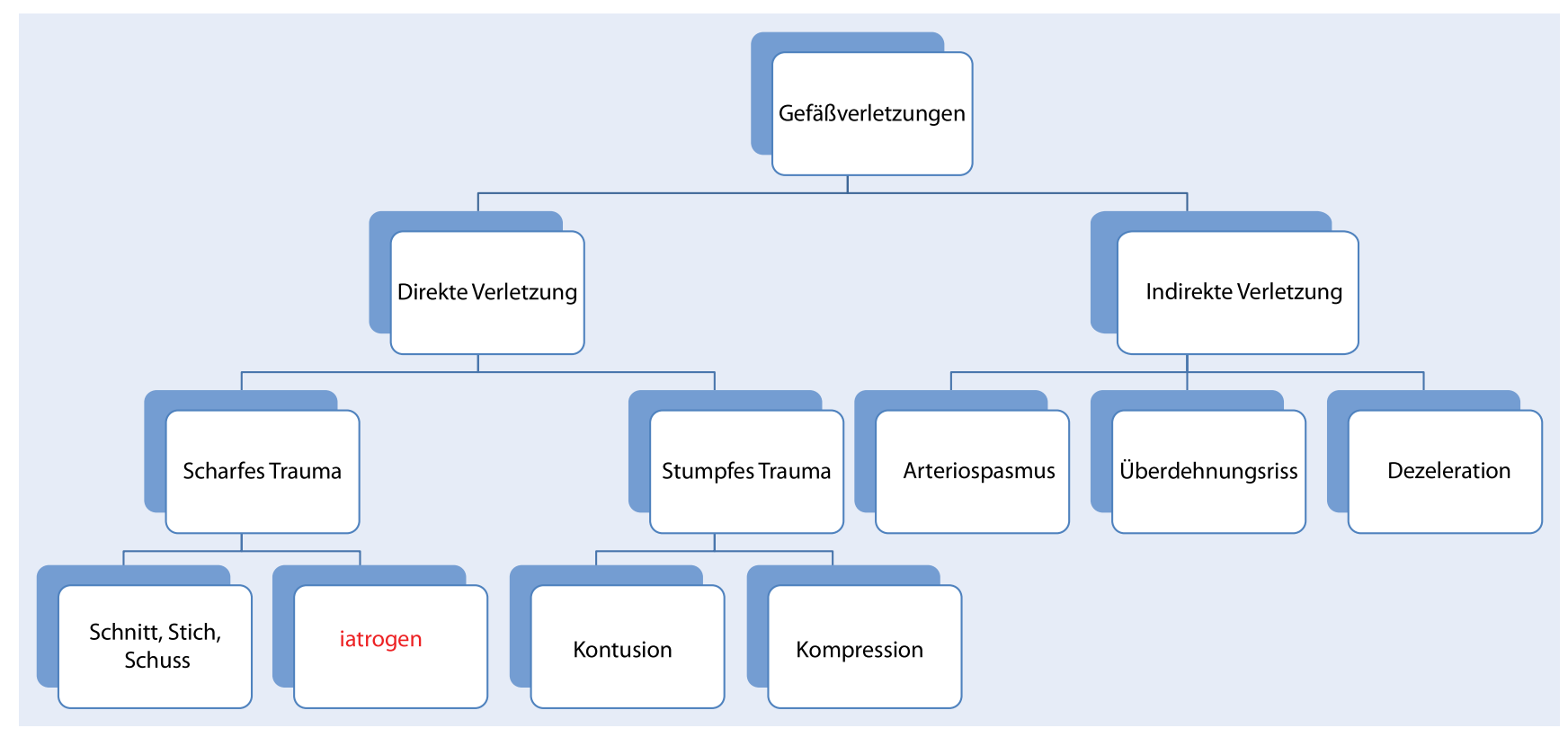

Abb. 1 A Klassifikation der Gefäßverletzungen nach Lindner und Vollmar [29]

Bei einer intraoperativen Blutung ist, so banal es klingen mag, die Kompressionstherapie mittels Finger oder Kompresse die erste Maßnahme, um sich einen Überblick über das Ausmaß der Verletzung zu machen, ggf. das Präparationsfeld für eine Gefäßausklemmung zu erweitern und entsprechende Instrumente (z. B. Cell Saver ${ }^{\circledast}$ ) zu organisieren. Die Wertigkeit der Autotransfusion mit Cell Saver ${ }^{\circ}$ wurde in einem systematischen Cochrane Review im Rahmen der Chirurgie u. a. rupturierter Aortenaneurysmen unlängst untersucht und eine Reduktion der Transfusion von Blutprodukten belegt [9].

\section{》) Die Kompressionstherapie mittels Finger oder Kompresse ist die erste Maßnahme}

Die unmittelbar intraoperativ durchführbare differenzierte Gerinnungsanalyse durch den Anästhesisten ist eine wichtige und wertvolle ergänzende Maßnahme. Nur bei zu erwartender arterieller Ausklemmung ist ggf. eine Heparingabe i.v. indiziert. Alternativ kann bei Arterienverletzungen auch selektiv Heparin mit einer Knopfkanüle in die beiden Arterienenden vor geplanter Reanastomosierung appliziert werden. Allgemein empfiehlt sich die intraoperative Verwendung einer Lupenbrille. Kenntnisse gefäßchirurgischer Nahttechniken sind hilfreich bzw. eine wichtige Voraussetzung für eine schnelle, suffiziente und andauernde Rekonstruktion von Gefäßverletzungen. Am Rande sei hier auf ein Aus- und Weiterbildungsdefizit in diesem Gebiet im Rahmen der aktuellen Weiterbildungsordnung mit „common trunk“ und früher Spezialisierung hingewiesen. Eine gefäßchirurgische Rotation ist aus unserer Sicht insbesondere für Chirurginnen/Chirurgen mit dem Schwerpunkt Tumorchirurgie und/oder Transplantationschirurgie sehr empfehlenswert. Der intraoperative Einsatz der Duplexsonographie oder Angiographie ist nicht vorgeschrieben, stellt aber aus eigener Erfahrung eine wichtige und optimierte Kontrolle der Rekonstruktion im Sinne der Qualitätskontrolle des technischen Erfolges und damit der Offenheitsrate dar.

Dem Chirurgen stehen zur intraoperativen Blutungskontrolle zahlreiche Methoden zur Verfügung. Insbesondere der bipolare Elektrokauter, eingeführt durch William T. Bovie und den Neurochirurgen Harvey Cushing im Jahr 1926, ist ein nützliches Instrument zur Blutstillung kapillarer arterieller Blutungen und kleiner venöser Blutungen. Der Argonbeamer hat den Vorteil, dass kein direkter Kontakt zum Gewebe vorliegen muss und wird v. a. bei parenchymatösen Blutungen oder großen Präparationsflächen eingesetzt. Für arterielle Blutungen und Blutungen größerer Venen ist er aus gefäßchirurgischer Sicht nicht geeignet, hier sollte primär die Gefäßnaht angewendet werden. Weiterhin existiert eine Reihe absorbierbarer hämostatischer Agenzien wie Gelatineschwämme, Zellulose, mikrofibrilläres Kollagen (MFC), fibrinhaltige Zweikomponentengewebekleber, Thrombin und Thrombin-Gelatin-Kombinationen. Auf die Beschreibung der unterschiedlichen Wirkungsmechanismen, der Vorund Nachteile sowie die Preisunterschiede kann aus Platzgründen nicht detailliert eingegangen werden.

Der in $\bullet$ Abb. 2 dargestellte Algorithmus kann bei der Wahl der Verfahren helfen.

\section{Arterielle Blutungen}

\section{Verletzungen der abdominellen Viszeralarterien}

Die Verletzung viszeraler Hauptstammarterien (Arteria mesenterica superior [AMS], Truncus coeliacus [TC]) im Rahmen abdomineller Operationen ist extrem selten. Die Inzidenz beträgt $0,01-$ $0,1 \%$ aller Gefäßverletzungen [10]. Bei unübersichtlichem Situs und lokal unkontrollierbarer Blutung kann nach 


\section{Böckler · A. Hyhlik-Dürr · M. Hakimi · T. Brenner · A. Ulrich · S. Hofer Management von Blutungen und Infektionen im Rahmen viszeralchirurgischer Operationen}

\author{
Zusammenfassung \\ Blutungen und Gefäßinfektionen sind \\ potenzielle Komplikationen im Rahmen \\ viszeralchirurgischer Operationen. Das \\ Management hängt von der Ausdehnung \\ und Lokalisation der Blutung ab und kann \\ von der Applikation von Hämostyptika \\ bis hin zur Gefäßnaht, Interposition oder \\ Ligatur reichen. Bei zweizeitigen Blutungen \\ verdrängen endovaskuläre Stentgraft- \\ Implantationen die klassischen kon- \\ ventionellen chirurgischen Verfahren zu- \\ nehmend. Infektionen von Gefäßen und \\ alloplastischen Implantaten werden immer \\ seltener. Die Diagnostik ist meist ver- \\ schleppt und das Management komplex \\ und langwierig. Die radikale Exzision, auto-
}

loge Rekonstruktion und Langzeitantibiose sind die Säulen der Therapie von Gefäßinfektionen. Patientenüberleben und Infektrezidivfreiheit sind die primären Therapieziele. Die Begleitmorbidität ist jedoch hoch. Aortoenterale Fisteln (AEF) sind mit einer Inzidenz von $<1,5 \%$ selten. Meistens treten sie als sekundäre Fisteln nach vorausgegangener offener oder endovaskulärer Aortenchirurgie auf. AEF besitzen eine hohe Morbidität und Mortalität von $40-100 \%$. Der direkte Fistelnachweis ist trotz moderner Bildgebung nach wie vor schwierig. Als Diagnoseverfahren besitzen die Computertomographie mit Kontrastmittelverstärkung (CTA) und die Fluorodeoxyglucose-Positronenemissions-
Computertomographie (FDG-PET-CT) bei Low-grade-Infekten die höchste Sensitivität (>90\%) und Spezifität (>80\%). Die Therapie besteht aus dem ein- bzw. zweizeitigen Verfahren mit Dünndarmteilresektion und anatomischer, ggf. extraanatomischer Aortenrekonstruktion. Die endovaskuläre Aortenrekonstruktion (EVAR) ist nur bei primären aortoenteralen Fisteln sowie bei Patienten mit sekundärer Fistel ohne Zeichen einer Infektion bzw. als Bridging-Verfahren im Notfall sinnvoll.

Schlüsselwörter

Komplikation - Chirurgie - Arterie - Vene .

Aortoenterale Fistel

\section{Management of bleeding and infections in the context of visceral surgery}

\section{Abstract}

Bleeding and vascular infections are serious potential complications during abdominal general surgery. The management of bleeding depends on the extent and localization and can range from the application of hemostatics to vascular sutures, interpositioning and ligatures. The use of prosthetic biomaterials implanted endoluminally or during open reconstruction permits palliation of potentially fatal conditions. The overall incidence of infections involving vascular prostheses is relatively low because of routine antibiotic prophylaxis prior to surgery, refinements in sterilization and packaging of devices and careful adherence to aseptic procedural and surgical techniques. When infections occur detection and definitive therapy of the vascular prosthesis are often delayed and the man- agement is complex and tedious. Infections involving vascular prostheses are difficult to eradicate and in general, surgical therapy is required often coupled with excision of the prosthesis. Keys to success include accurate diagnostics to identify the organism and extent of graft infections, specific long-term antibiotic therapy and well-planned surgical interventions to excise and replace the infected graft and sterilize the local tissue. Regardless of the technique used to eradicate graft infections, success is measured by patient survival, freedom from recurrent infection and patency of revascularization. Even when treatment is successful, the morbidity associated with vascular graft infections is considerable. Aortoenteric fistulas (AEF) are a rare (incidence $<1.5 \%$ ) but often fatal compli- cation. Primary diagnosis of AEF remains difficult. Computed tomography (CT) and fluorodeoxyglucose positron emission tomography CT (FDG-PET-CT) are the diagnostic tools of choice. Therapy consists of an urgent individualized interdisciplinary surgical approach with primary axillofemoral bypass and secondary prosthesis explantation or in situ replacement and subsequent bowel resection. Endovascular aortic repair (EVAR) is reserved for primary aortoenteric fistulas in patients with no signs of infection or in emergency cases as a bridging method.

\section{Keywords}

Complication · Surgery · Artery · Vein . Aortoenteric fistula
Spaltung des Omentum minus und beider Zwerchfellschenkel eine subdiaphragmale bzw. supratrunkale Aortenklemmung notwendig und sinnvoll sein. Als Gefäßrekonstruktionen kommen je nach Ausdehnung die Direktnaht, die Patchplastik oder das Interponat infrage. Bei potenziell bakteriell kontaminiertem Situs sollte auf autologes Ersatzmaterial, z. B. mit Vena saphena magna oder Rinderperikard, zurückgegriffen werden. Alloplastisches Material (Dacron, Polytetrafluorethylen [PTFE]) führt zum Infekt (siehe im Folgenden unter „Infektionen“) und ist wegen der Blutungsgefahr kontraindiziert.

Sollte eine alloplastische Rekonstruktion aus Gründen eines Kalibermismatches zwischen Vene und zu rekonstruierendem Gefäß notwendig werden, ist auf eine sorgfältige Retroperitonealisierung $\mathrm{zu}$ achten, um prothetoenterale Fisteln und sekundäre Protheseninfekte zu vermeiden. In scheinbar ausweglosen Situationen und lebensbedrohlichem Schockzustand kann die primäre Ligatur des TC oder der AMS gerechtfertigt sein. Eine simultane Unterbindung verbietet sich [11]. Ob die gastro- duodenale Arkade zwischen AMS und TC zur Perfusion von Leber und Magen bei Ligatur des TC ausreicht, ist schwer bzw. nicht zu prognostizieren. Eine intraoperative Duplexsonographie der Leberpforte kann hier weiterhelfen. Eine zeitnahe Second-look-Operation $(<48 \mathrm{~h})$ ist notwendig, um potenzielle Organischämien rechtzeitig zu erkennen.

\section{Arrosionsblutungen}

Arrosionsblutungen von Gefäßanastomosen entstehen meist im arteriellen Gefäßgebiet im Rahmen 


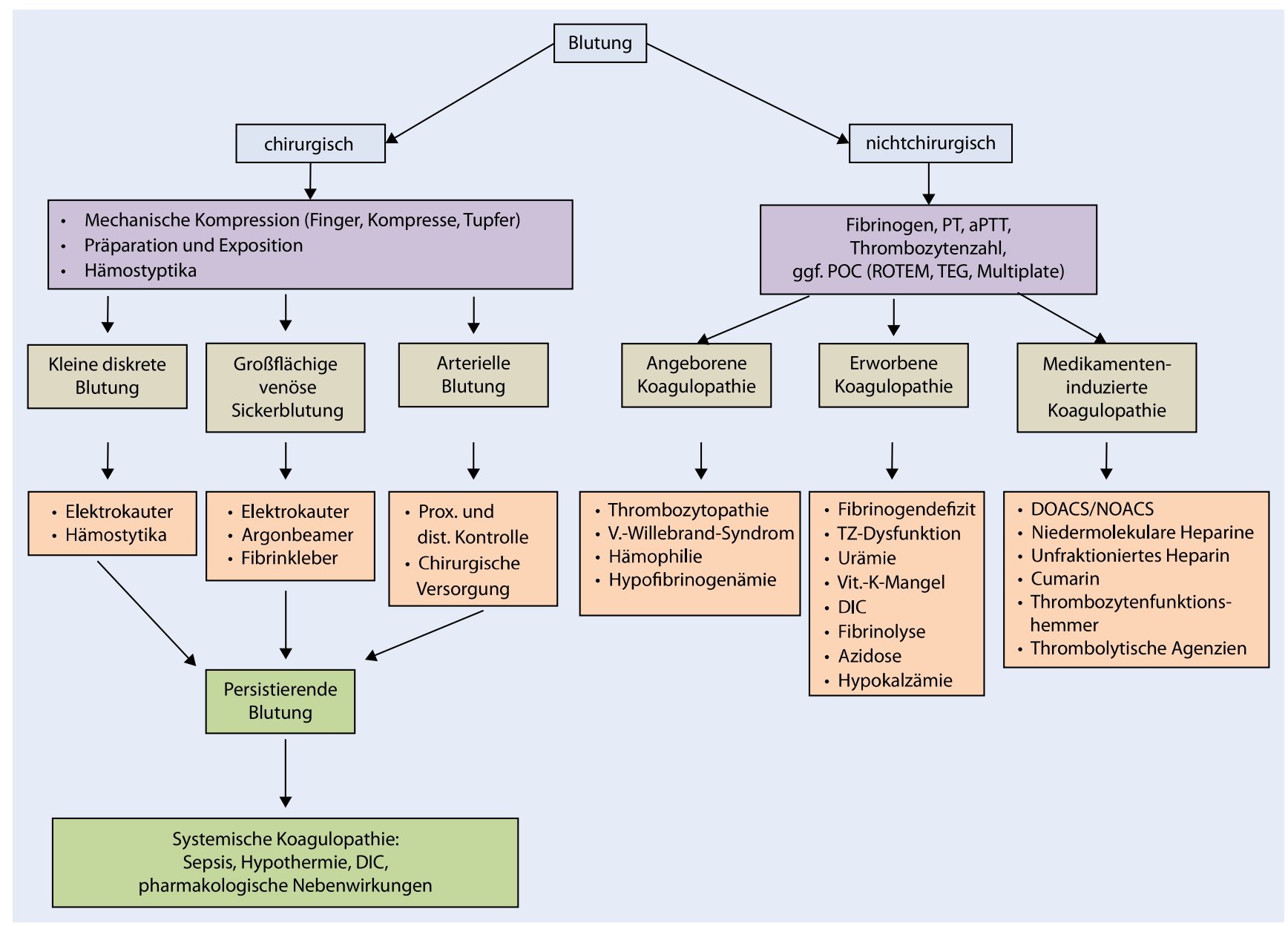

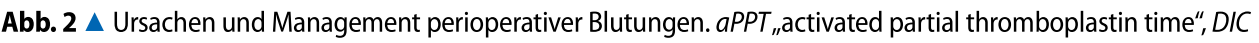
"disseminated intravascular coagulation", DOACS ,direct-acting oral anticoagulants", NOACS , new oral anticoagulants", POC Point-of-care-Verfahren, $P T_{\text {" }}$ prothrombin time", TEG Thromboelastogramm, TZThrombozyten

akuter und chronischer Infekte, d. h. intraoperativen Keimkontaminationen in Folge einer Durchwanderungsperitonitis, Hohlorganperforation oder Anastomoseninsuffizienz. Die endovaskuläre Schienung mit gecoverten Stentgrafts kann lebensrettend sein, ist jedoch als Bridging einzustufen und muss definitiv konvertiert werden, $d$. $h$. Explantation des Stents und autologe Gefäßrekonstruktion müssen in der Folge durchgeführt werden. Ein Belassen des gecoverten Stents in einem potenziell infizierten Situs kommt einer Palliativsituation gleich.

\section{Blutungen aus}

\section{Anastomosenaneurysmen}

Selten stellen rupturierte Anastomosenaneurysmen die Ursache für abdominelle Blutungen dar. Sie treten im aortoiliakalen Abschnitt nach durch- schnittlich 6 Jahren in 1,4-4\% der Fälle auf. Gefäßanastomosen in infizierten Regionen stellen einen Risikofaktor für eine spätere Aneurysmaformation dar. Zur Diagnosestellung steht v. a. hinsichtlich Verfügbarkeit und Praktikabilität die computertomographische Angiographie (CTA) als Mittel der 1. Wahl zur Verfügung. Obwohl Bastounis et al. nachgewiesen haben, dass sich Duplexsonographie, Magnetresonanztomographie (MRT) und CT in ihrer Sensitivität nicht unterscheiden, ist die CTA die Methode der 1. Wahl zur Diagnostik.

Infizierte Aneurysmen bedürfen oft komplexer rekonstruktiver Maßnahmen mit Homograft oder Venafemoralis-superficialis-Ersatz (sog. „deep vein“). Bei Blutungen nichtinfizierter rupturierter Anastomosenaneurysmata hat die endovaskuläre Therapie die konventionelle Chirurgie in vielen Kliniken ersetzt. Sowohl interventionelle Radiologen als auch endovaskulär erfahrene Gefäßchirurgen haben mit gecoverten Stents in Single-Center-Serien mit 10 bis 60 Patienten in den letzten Jahren 100\% technischen Erfolg publiziert [12-14].

\section{Venöse Blutungen}

Im Rahmen von Tumorresektionen der kolorektalen und hepatobiliären Chirurgie sind Verletzungen intraabdomineller Venen möglich. Gefährdet sind v. a. die Vena portae; Mesenterialvenen, Beckenvenen und die untere Hohlvene.

Venöse Blutungen sind aus gefäßchirurgischer Sicht anspruchsvoller und bei großlumigen Gefäßen aus Sicht der Autoren gefährlicher. Dies liegt an den zahlreichen zufließenden Ästen und dem komplexen Ein- und Ausstromnetzwerk, was die proximale und distale 


\begin{tabular}{|c|c|}
\hline \multirow{2}{*}{$\begin{array}{l}\text { Zeitpunkt des Auftretens eines In- } \\
\text { fektes }\end{array}$} & Früher Infekt: $<4$ Monate \\
\hline & Später Infekt: > 4 Monate \\
\hline \multirow{3}{*}{$\begin{array}{l}\text { Postoperative Wundheilungsstörung } \\
\text { (Klassifikation nach Szilagyi) }\end{array}$} & Grad I: Cellulitis \\
\hline & Grad II: Infektion des Subkutangewebes \\
\hline & Grad III: Infektion des Gefäßes/Grafts \\
\hline \multirow{8}{*}{$\begin{array}{l}\text { Ausmaß der Graft-Infektion (Klassi- } \\
\text { fikation nach Bunt) }\end{array}$} & Periphere Graft-Infektion: \\
\hline & P0 = in einer Körperhöhle (z. B. Pfortaderersatz) \\
\hline & P1 = außerhalb der Körperhöhle (z. B. Leiste) \\
\hline & P2 = abdominell-peripherer Bypass (z. B. aortofemoral) \\
\hline & P3 = Patchplastik (z. B. Femoralispatchplastik) \\
\hline & Prothetoenterische Erosion \\
\hline & Prothetoenterische Fistel \\
\hline & Septische Aortenstumpfarrosionsblutung \\
\hline
\end{tabular}

Gefäßkontrolle schwieriger macht. Die Dünnwandigkeit von Venen und die chirurgische Manipulation können das Verletzungsausmaß schnell vergrößern. Kleine venöse Blutungen können durch die Applikation hämostyptischer Agenzien oder „geduldiger“ Kompression mit Kompressen oder Bauchtüchern (ggf. temporär als Packing) erfolgreich kontrolliert werden. Ligaturen von Venen bleiben im Gegensatz zu Arterien aufgrund ihres Kollateralnetzwerkes oft folgenlos und können somit liberaler durchgeführt werden.

\section{》) Venöse Blutungen werden hinsichtlich des Blutverlustes häufig unterschätzt}

Die Mortalität der V.-cava-inferior-Verletzung im Rahmen iatrogener Begleitverletzungen hat bei unverzüglicher Versorgung eine bessere Prognose als die traumatische Verletzung der unteren Hohlvene, die mit einer Sterblichkeit von $50 \%$ assoziiert ist [15]. Primäres Ziel bei der Verletzung großer Venen ist die Blutungskontrolle und sekundär, wenn möglich, die Rekonstruktion. Ggf. ist die Ligatur notwendig und indiziert, die in der Regel durch venöse Kollateralkreisläufe gut kompensiert wird. Primär ist die übersichtliche Präparation, Exploration und gute Übersicht eine wichtige Grundvoraussetzung. Technisch stehen die temporäre Tamponade, die Ligatur oder Rekonstruktion mittels Direktnaht, Patchplastik oder Interponat als alternative Verfahren zur Verfügung. Venöse
Blutungen werden hinsichtlich des zu erwartenden Blutverlustes häufig unterschätzt, sodass eine frühzeitige Verwendung des Cell Saver empfohlen ist.

\section{Management postoperativer Blutungen}

Unmittelbar postoperative Blutungen können eine chirurgische Ursache im Sinne der chirurgischen Nachblutung haben oder einer Gerinnungsstörung zugrunde liegen. Erste Symptome sind Hypotension und Tachykardie (Schockparameter), zurückgehende Urinausscheidung, Abfall des Hämoglobinwertes und als Spätzeichen ggf. klinisch apparente Hämatomentwicklung („pralles Abdomen“). Allgemeine Maßnahmen unabhängig der genannten Ätiologie sind in Anhängigkeit von der hämodynamischen Stabilität Normothermie, aktuelle Bestimmung des kleinen Blutbildes, der Gerinnung, ggf. aber auch eine erweiterte Gerinnungsanalyse (sog. "point of care testing“) inkl. Fibrinogen und Thromboelastometrie (ROTEM ${ }^{\circledR}$-Analyse; [5]) und zeitgleich die optionale Bestellung von Blutprodukten zur Transfusion. Die Durchführung eines Thromboelastogrammes (TEG) bei akuter Blutung kann sinnvoll sein, da gezeigt werden konnte, dass ein pathologisches TEG mit einer erhöhten Mortalität assoziiert ist [16].

Gemeinsam mit Anästhesisten und der evtl. notwendigen i.a. Volumensubstitution die Transfusion von Erythrozytenkonzentraten und GeIntensivmedizinern ist deshalb neben rinnungsfaktoren $\mathrm{zu}$ besprechen und $\mathrm{zu}$ indizieren. Vor einer „blinden“ Antagonisierung des intraoperativ gegebenen Heparins mit Protamin wird zunächst abgeraten, da es sich selten um eine heparininduzierte Blutung handelt. Am Rande sei in diesem Zusammenhang auf die kumulative Wirkung fraktionierter und unfraktionierter Heparine bei niereninsuffizienten Patienten hingewiesen.

Parallel kann eine erste orientierende Ultraschalluntersuchung erfolgen, im Zweifelsfalle eine kontrastmittelunterstützte CT-Angiographie (cave: Nierenfunktion) indiziert sein. Wenn der Patient trotz ausgeglichenem Gerinnungsstatus laborchemische und klinische Zeichen der Blutung zeigt, sollte die notfallmäßige Indikation zur Revision früh und großzügig gestellt werden.

\section{Infektionen}

Die publizierte Inzidenz von Gefäßinfektionen, sowohl bei nativen Gefäßen als auch bei Gefäßprothesen, beträgt $0,2-$ $5 \%$. Das Potenzial der Entstehung eines späten, oft Monate nach dem Ersteingriff auftretenden Infektes wird oft unterschätzt.

Einflussfaktoren sind v. a. der Implantationsort, die Indikation und die Immunabwehr des Patienten. Die Infektion prothetischer Implantate und Endoprothesen wird klinisch in Abhängigkeit von dem Zeitpunkt des Auftretens, von dem Grad der Wundheilungsstörung (Klassifikation nach Szilagyi) und dem Ausmaß der Graft-Beteiligung (Bunt-Klassifikation) eingeteilt (• Tab. 2) Frühe Infektionen sind meist mit SzilagyiGrad-III-Infekten assoziiert, treten in der Regel 1 bis 3 Monaten nach Entlassung auf und betreffen v. a. extrakavitäre, also periphere Implantate. Gefäß- und Implantatinfektionen im Abdomen nach viszeralchirurgischen Eingriffen oder zentralen gefäßchirurgischen Rekonstruktionen gehören meist zu den späten Infekten $(>4$ Monate). Selbst oberflächliche Wundinfekte Grad I und II nach Szilagyi können dabei zu Prothesenspätinfekten führen. Das Keimspektrum sind v. a. Staphylococcus epidermidis, seltener Candida [17]. 


\begin{tabular}{|l}
\hline Infobox 1 Einflussfaktoren auf \\
die bakterielle Kontamination von \\
Gefäß-/Protheseninfekten \\
\hline Perioperative Faktoren \\
- Prolongierter Krankenhausaufenthalt \\
- Infektion im operativen Zugangsbereich \\
- Kürzlich zurückliegender perkutaner \\
arterieller Zugang \\
- Notfalloperation \\
- Rezidiveingriff \\
- Lange bzw. verlängerte Operationszeiten \\
- Simultane Operationen des Gastro- \\
intestinal- und Urogenitaltraktes \\
- Postoperative Wundkomplikationen \\
(Wundrandnekrose, Lymphozele) \\
Patientenassoziierte Faktoren/geschwächte \\
Immunabwehr \\
- Maligne Grunderkrankung \\
- Lymphoproliferative Erkrankungen \\
- Autoimmunkrankheiten \\
- Kortikosteroidtherapie \\
- Chemotherapie \\
- Mangelernährung \\
- Diabetes mellitus \\
- Chronische Niereninsuffizienz, Dialyse \\
- Lebererkrankungen/-zirrhose \\
\hline
\end{tabular}

\section{Ätiologie}

Die Präsenz eines Fremdkörpers potenziert die Infektiosität von Bakterien. Das Risiko steigt mit der Virulenz der Keime sowie vor allem bei Immunsuppression. Pathogenetisch kommt es zur Adhäsion von Bakterien auf der Graft- oder Stentgraft-Oberfläche, der Bildung von Mikrokolonien innerhalb eines bakteriellen Filmes, der Aktivierung von Abwehrmechanismen (Zytokinfreisetzung, neutrophile Chemotaxis, Komplementaktivierung) und einer inflammatorischen Antwort im Perigraft-Gewebe und der Anastomose zwischen Graft und Arterie. Wichtig ist bei der Prothesenwahl zu wissen, dass die bakterielle Adhäsion auf Polyester 10- bis 100-mal größer ist als bei PTFE.

Die Exposition von Biomaterialien bzw. alloplastischen Implantaten mit Mikroorgansimen/Bakterien können durch vier Mechanismen zu einer klinischen Infektion führen:

- perioperative Kontamination über die chirurgische Wunde (insbesondere beim Platzbauch),
- Besiedelung im Rahmen einer Bakteriämie (Pneumonie, Harnwegsinfekt, Kathetersepsis, Endokarditis),

- mechanische Arrosion mit dem Dünn/-Dickdarm, aber auch der ableitenden Harnwege (Inzidenz 0,4$2 \%)$ oder durch einen

- benachbarten lokalen Entzündungsprozess.

Daraus ergeben sich die wichtigsten chirurgisch-strategischen Maßnahmen zur Vermeidung von Gefäß- und Protheseninfektionen: aseptische Chirurgie, perioperative leitliniengerechte Antibiotikaprophylaxe, subtile Weichteildeckung der Gefäßrekonstruktionen, und wenn möglich, die Durchführung der Operation im infekt- bzw. bakteriämiefreien Intervall.

Risikofaktoren, die zu einer bakteriellen Kontamination und Gefäß- bzw. GraftInfektion führen sind in $\bullet$ Infobox 1 aufgeführt und unterscheiden grob perioperative Faktoren und patientenassoziierte Faktoren. Notfalloperationen, lange Operationszeiten, maligne Grunderkrankungen, Chemotherapie, chronische Niereninsuffizienz, Diabetes mellitus seien in Zusammenhang mit dem Fokus dieses Artikels auf viszeralchirurgische Eingriffe besonders hervorgehoben.

\section{Diagnostik und Therapie von Infekten}

Die kontrastmittelunterstütze CTA ist die präferierte bildgebende Untersuchungsmethode bei postoperativen Gefäß- und Protheseninfekten, nicht nur aufgrund ihrer schnellen, einfachen und ubiquitär verfügbaren Durchführung wegen. Indirekte Zeichen des Infektes sind Flüssigkeitsansammlung und Lufteinschlüsse um die Prothese, wobei unmittelbar postoperative Lufteinschlüsse noch normal sein können, aber spätestens 2 bis 3 Monate postoperativ nicht mehr nachweisbar sein sollten. Insbesondere die CT-gesteuerte Punktion mit Gewinnung mikrobiologischer Abstriche und ggf. Drainageeinlage ist eine minimalinvasive effektive Maßnahme, ersetzt aber die Operation im Falle des Infektnachweises nicht.

Die MRT kann in den unterschiedlichen T1- und T2-Gewichtungen Flüssig- keiten von Fibrosierungen besser unterscheiden und stellt eine gute Alternative zur CTA v. a. bei niereninsuffizienten Patienten dar. Die Leukozytenszintigraphie spielt heutzutage keine Rolle mehr in der Diagnostik von Gefäßinfekten.

Das operative Management beinhaltet in Abhängigkeit von Manifestation, Ausdehnung des Infektes und zugrunde liegendem Keimspektrum mehrere Optionen. Eine tabellarische Übersicht gibt $\bullet$ Tab. 3. Eine detaillierte Beschreibung ist aus Platzgründen in diesem Artikel nicht möglich. Es sei auf folgende Literaturstellen verwiesen [18].

In Kürze seien folgende Hinweise gegeben: Ein Belassen kontaminierter Gefäße und Prothesenimplantate sowie ein partieller Prothesenausbau ist sehr kritisch zu sehen und bleibt Ausnahmesituationen vorbehalten. Wenn technisch möglich und dem Patienten zumutbar, ist der komplette Ausbau, die Kürettage des Bypasslagers und die antiseptische Spülung des Situs mit anschließender Rekonstruktion in Abhängigkeit vom Infektionsgrad zu raten. Eine antibiotikabehandelte Prothesenimplantation kann bei begrenzten, sog. Low-grade-Infekten mit z. B. S. epidermidis, in Erwägung gezogen werden. Kryopräservierte arterielle Allografts („Leichenarterien“) sind limitiert in ihrer Verfügbarkeit und in ihrer aneurysmatischen Degeneration im Langzeitverlauf. Bei Verwendung der körpereigenen Vene, meist Vena saphena magna oder Vena femoralis superficialis, ist ein ausreichendes Kaliber (>5 mm) bei der Verwendung im viszerorenalen Arteriensegment wichtige Voraussetzung für die Offenheitsrate. Ein Kalibermismatch von mehr als $6 \mathrm{~mm}$ ist potenziell problematisch.

\section{Aortoenterale Fistel}

Die aortoenterale Fistel (AEF) ist eine Rarität und stellt eine häufig fatale aortale Komplikation dar. Sie erfordert eine interdisziplinäre Zusammenarbeit zwischen Nuklearmedizinern sowie Gefäß- und Viszeralchirurgen. Aufgrund der Seltenheit der Erkrankung existieren keine kontrollierten Studien [19]. Die vorliegenden Erkenntnisse beruhen auf der Auswertung von Einzelfall- 


\begin{tabular}{|c|c|c|c|}
\hline Behandlungsoption & Manifestation & Ausdehnung des Infektes & Mikrobiologie \\
\hline $\begin{array}{l}\text { Lokale Therapie, Graft (Vene, evtl. } \\
\text { auch Prothese) in situ belassen }\end{array}$ & Frühe Infektion, keine Sepsis & $\begin{array}{l}\text { Kein Dacron, Graft-Hauptkörper ohne } \\
\text { Anastomosenbeteiligung, segmental }\end{array}$ & $\begin{array}{l}\text { Grampositive Keime, Staphy- } \\
\text { lococcus }\end{array}$ \\
\hline Ersatzlose Graft-Explantation & $\begin{array}{l}\text { Graft-Thrombose, vitale Organe/Extremi- } \\
\text { täten, adäquater Kollateralkreislauf }\end{array}$ & Jegliche (P0-P3, siehe $\bullet$ Tab. 2) & Jegliches Keimspektrum \\
\hline \multicolumn{4}{|l|}{ Explantation und Ex-situ-Bypass } \\
\hline -Simultan & $\begin{array}{l}\text { Instabiler Patient, AEE/AEF, Hämorrhagie, } \\
\text { schwere Sepsis }\end{array}$ & Invasive Infektion & Jegliches Keimspektrum \\
\hline -Zweizeitig & $\begin{array}{l}\text { "Stable patient",",mild sepsis", AEE/AEF, } \\
\text { „no active bleeding" }\end{array}$ & Invasive Infektion & Jegliches Keimspektrum \\
\hline \multicolumn{4}{|l|}{ In-situ-Rekonstruktion } \\
\hline -Prothese (alloplastisch) & Keine Sepsis, keine AEE/AEF & Biofilm-Infektion, segmental & $\begin{array}{l}\text { Staphylococcus epidermidis, } \\
\text { gramnegative Keime }\end{array}$ \\
\hline -Körpereigener Vene (autolog) & $\begin{array}{l}\text { Keine Sepsis, keine AEE/AEF, schwere } \\
\text { okklusive Gefäßsklerose }\end{array}$ & $\begin{array}{l}\text { Invasive oder Biofilm-Infektion, diffus } \\
\text { oder segmental }\end{array}$ & Nicht bei Pseudomonas \\
\hline
\end{tabular}

studien, Fallserien und systematischen Literaturanalysen. Durch eine frühzeitige Diagnostik und entsprechende Therapie ist es möglich, die Prognose zu verbessern [20]. Pathogenetisch lassen sich eine primäre und eine sekundäre Fistel unterscheiden. Bei letzteren geht ein operativer aortaler Gefäßersatz durch offene oder endovaskuläre Rekonstruktion voraus. Primäre aortale Fisteln zeigen eine Häufigkeit von $0,02-0,07 \%$ [21], sekundäre sind mit einer Inzidenz von $0,4-2,4 \%$ [22] nach konventionellem Aortenersatz und $0,7 \%$ nach endovaskulärer aortaler Rekonstruktion (EVAR) vergesellschaftet [8].

Bei primären Fisteln kommt es durch Darmulzerationen, perforierte Divertikel, Fremdkörperperforationen oder tumorbedingt zum Kontakt des Darminhalts mit dem Gefäßsystem und zur Ausbildung einer Fistel. Von vaskulärer Seite fördern mykotische oder rupturierte Aneurysmen, aber auch die Entzündung im Sinne der Aortitis sowie die ständige mechanische Belastung durch die Pulsation die Entstehung einer primären aortalen Fistel. Sekundäre aortale Fisteln werden durch eine Minderdurchblutung des betroffenen Darmabschnitts oder durch eine mögliche operative Denervierung oder Deserosierung während des Primäreingriffs mit oft nach Jahren (im Mittel 2 bis 5 Jahre) folgender Perforation begünstig. Nahtaneurysmen (Aneurysma falsum) sowie ein zentraler Protheseninfekt begünstigen die Ausbildung sekundärer aortaler Fisteln. Der mit $70 \%$ am häufigsten betroffene Darmabschnitt ist das Duodenum mit seiner retroperitoneale Lage. Das Jejunum ist mit $12 \%$ am zweithäufigsten betroffen, Ileum und Kolon zeigen Häufigkeiten unter $10 \%$. Der Magen als seltener Ursprung intestinaler Fisteln zeigt eine Inzidenz $<5 \%$. Prinzipiell kann sich eine aortoenterale Fistel mit jedem Abschnitt des Gastrointestinaltraktes ausbilden.

Bei einer Mortalität der nicht behandelten aortoenteralen Fisteln von $100 \%$ kann die Prognose einzig durch eine strukturierte und schnelle Diagnostik sowie interdisziplinäre individuelle Therapie verbessert werden.

\section{Diagnostik}

Die spezifische Diagnostik aortoenteraler Fisteln stellt weiterhin eine große Herausforderung dar. Laboruntersuchungen zeigen in mehr als zwei Dritteln der ausgewerteten Fälle ( $>70 \%$ ) unspezifische Laborveränderungen mit Anstieg der Leukozyten oder des C-reaktiven Proteins (CRP). Eine direkte Darstellung der aortoenteralen Fistel (AEF) mithilfe der Gastroduodenoskopie wird durch die Lokalisation in der Pars III und IV des Duodenums erschwert und gelingt nur in einem Drittel der Fälle [23].

$>$ Höchste Aussagekraft besitzt aktuell die CT mit Kontrastmittelverstärkung (CTA).

Hiermit gelingt der Nachweis einer aortoenteralen Fistel mit $94 \%$ Sensitivität und $85 \%$ Spezifität. Die Genauigkeit wird im Vergleich der Studien mit $79 \%$ angegeben [24]. Durch die flächendeckende Verfügbarkeit und die minimale Invasivität ist die kontrastmittelverstärkte CT das Diagnostikum der Wahl und wird in seiner Genauigkeit nur von der Laparotomie (91\%) übertroffen.

Der direkte Fistelnachweis durch sichtbaren Kontrastmittelübertritt gelingt jedoch nur in Einzelfällen. Indirekte Zeichen wie pathologische Lufteinschlüsse oder Infektkriterien mit perivaskulären Flüssigkeitsansammlungen, lokale Gefäßwandverdickung oder die Ausbildung von Pseudoaneurysmen werden dagegen häufig beobachtet. In einzelnen Fällen mit chronischen Infekten an Grenzflächen zwischen Darm und Gefäßprothesen (Low-grade-Infekt) reduziert sich die Sensitivität auf 55\% [25]. Klinisch kann es durch sekundäre aortoenterale Fisteln bei dieser Infektkonstellation zu rezidivierenden septischen Embolien als führendes klinisches Symptom kommen, ohne dass es mit der konventionellen Diagnostik gelingt, den Nachweis einer aortoenteralen Fistel zu erbringen. Die Fluorodeoxyglucose-Positronenemission stomographie(FDG-PET)-CT weist eine Sensitivität und Spezifität von 91 bzw. $95 \%$ auf [26].

\section{Therapie}

Die möglichen Therapiealternativen primärer und sekundärer Fisteln beinhalten die konventionell offene Operation mit variablen Möglichkeiten 


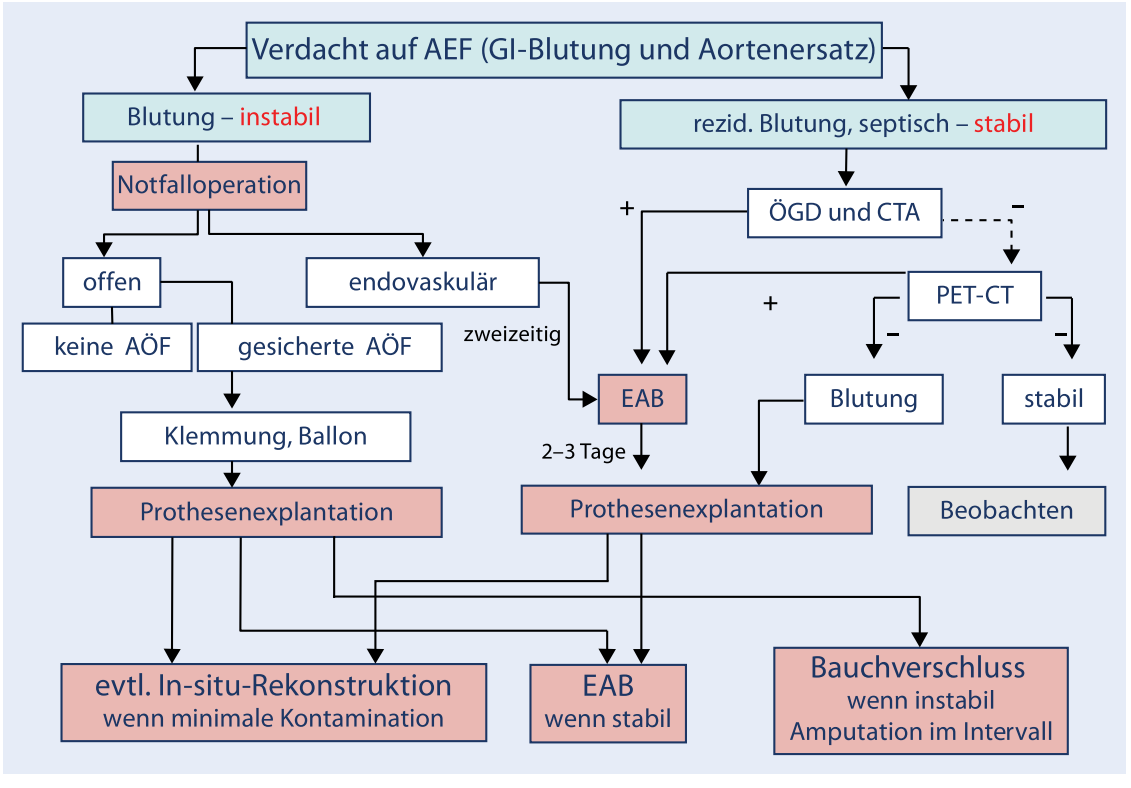

Abb. $3 \Delta$ Algorithmus zur differenzierten Diagnostik und individuellen Therapie bei Verdacht auf eine AÖF. AÖF aortoösophageale Fistel, CT Computertomographie, CTA computertomographische Angiographie, EAB extraanatomsicher Bypass, Gl gastrointestinal, ÖGD Ösophagogastroduodenoskopie, PET Positronenemissionstomographie

der In-situ-Rekonstruktion, den zweizeitigen Prothesenausbau mit primärer extraanatomischer Revaskularisation und im Intervall von 2 bis 3 Tagen folgender Aortenligatur (• Abb. 3). Als dritte Therapieoption steht die endovaskuläre Therapie zur Verfügung.

Abhängig von der gewählten Therapie besteht eine Mortalität zwischen 20 und $100 \%$. Ursächlich für das Versterben der schwerkranken Patienten sind geordnet nach Häufigkeit

- der hämorrhagische Schock,

- der septische Schock mit Multiorganversagen,

- kardiale Todesursachen,

- der Verblutungstod nach Aortenstumpfligatur bei Insuffizienz.

Die konservative Therapie aortaler Fisteln ist keine Alternative zur chirurgischen Therapie und zeigt eine $100 \%$ ige fistelassoziierte Mortalität.

Bei eingeschränkter Vergleichbarkeit der wenigen verfügbaren Studien zeigt die zweizeitige, extraanatomische Revaskularisation mit primärer Anlage eines axillobifemoralen Bypass und sekundärem Prothesenausbau mit Durchführung der Aortenstumpfligatur bei 22 $31 \%$ der Fälle die geringste Mortalität. Es muss jedoch in Betracht gezogen werden, fizierten Aortenprothese mit simultanem Ersatz durch allogenes oder alloplastisches Gefäßersatzmaterial zeigt in der aktuellen Analyse von Bergqvist eine Mortalität von 38-47\%. Der direkte Verschluss der infizierten Aorta mit Direktnaht oder Patch ist mit einer sehr hohen Mortalität von über $60 \%$ verbunden und ist demnach keine Alternative.

Seit den 1990er Jahren ist die endovaskuläre Therapie eine anerkannte Alternativmethode zur Behandlung elektiver und rupturierter abdomineller Aortenaneurysmen. Wegen der hohen Morbidität und Mortalität von bis zu $33 \%$ bei der offenen Versorgung aortoenteraler Fisteln wurden erste Versuche unternommen, AEF mittels EVAR zu therapieren. Dadurch kann eine schnelle Ausschaltung der Blutung erfolgen, ohne dass das hochentzündete Gebiet mit allen möglichen Komplikationen der offenen Chirurgie tangiert werden muss. Allerdings kann durch die EVAR nur die Blutung gestoppt und die distale Perfusion aufrechterhalten werden. Der septische Herd, also die Verbindung von Darm und Aorta, wird nicht therapiert. Hierdurch kommt es in der Regel zu erneuten oder persistierenden Infekten mit folgender Sepsis in bis zu $70 \%$ der Fälle. Es konnte gezeigt werden, dass sekundäre Fisteln im Gegensatz zu primären aortoenteralen Fisteln nach EVAR ein 3-fach höheres Risiko einer persistierenden Infektion bzw. Rezidivinfektion besitzen. In einem systematischen Review von Antoniuo et al. ([21]; 1990 bis 2008, 33 Publikationen, 41 Patienten) konnte gezeigt werden, dass der präoperative Infektstatus das spätere Outcome beeinflusst. Die Hämodynamik des Patienten spielte in dieser Studie keine Rolle, wobei klinische Zeichen der Infektion mit Fieber und ein auffälliges Labor die Morbidität und Mortalität nach endovaskulärer Behandlung aortoenteraler Fisteln statistisch signifikant negativ beeinflussen. Eine Langzeitantibiose wird empfohlen, kann jedoch eine drohende Sepsis als häufigste Todesursache nach EVAR bei AEF nicht sicher verhindern.

Die endovaskuläre Therapie ist demnach eine Alternative für Patienten, die für eine offene Operation aufgrund der Morbidität nicht geeignet sind 
(Notfall-EVAR als sog. „Bridging“), bei primären aortoenteralen Fisteln und [29] bei stabilen Patienten mit sekundären AEF, die keinen Hinweis auf Septikämie mit positiver Klinik (Fieber) und Labor zeigen. Für alle anderen Patienten muss EVAR bei aortoenteralen Fisteln als ein reines Bridging-Verfahren bis zur definitiven offenen Versorgung gesehen werden.

\section{Fazit für die Praxis}

- Blutungen und Infektionen von Gefäßen und Gefäßimplantaten im Rahmen viszeralchirurgischer Operationen sind seltene Komplikationen, die allerdings in ihrem Management komplex und mit einer erhöhten Morbidität und Mortalität assoziiert sind.

- Diagnostik und Therapie sind aufgrund eines multimodalen Angebotes (Ultraschall, CTA, MRT, PET-CT sowie konventionelle Chirurgie vs. endovaskuläre Therapie) anspruchsvoll geworden. Interdisziplinäre Zusammenarbeit mit Radiologen (Bildgebung, ggf. Intervention) und Anästhesisten (Gerinnungsmanagement) sind dabei sehr wichtig.

- Der „moderne" Gefäßchirurg, der endovaskulär und konventionell gleichermaßen ausgebildet ist, ist ein wichtiger Partner in diesen klinischen Ausnahmesituationen und sollte in der heutigen Zeit, in der eine fachübergreifende chirurgische Expertise aus einer Hand meist nicht mehr verfügbar ist, frühzeitig in das Behandlungskonzept eingebunden werden.

- Prävention, frühzeitige Diagnostik, Interdisziplinarität, ,"Entscheidungsfreudigkeit" und schnelle Blutstillung bzw. konsequente Infektsanierung sind die entscheidenden Säulen, die das Überleben des Patienten sichern.

\section{Korrespondenzadresse}

\section{Prof. Dr. D. Böckler}

Klinik für Gefäßchirurgie und Endovaskuläre Chirurgie,

Universitätsklinikum Heidelberg Im Neuenheimer Feld 110, 69120 Heidelberg dittmar.boeckler@med.uni-heidelberg.de

\section{Einhaltung ethischer Richtlinien}

Interessenkonflikt. D. Böckler, A. Hyhlik-Dürr, M. Hakimi, T. Brenner, A. Ulrich und S. Hofer geben an, dass kein Interessenkonflikt besteht.

Dieser Beitrag beinhaltet keine Studien an Menschen oder Tieren.

\section{Literatur}

1. Hackert T, Schneider L, Büchler MW (2015) Current state of vascular resections in pancreatic cancer surgery. Gastroenterol Res Pract 2015:120207

2. Daniels M, Merkel S, Agaimy A et al (2015) Treatment of perforated colon carcinomas-outcomes of radical surgery. Int J Colorectal Dis 30(11):15051513

3. Schwarzbach MH, Hormann Y, Hinz U et al (2006) Clinical results of surgery for retroperitoneal sarcoma with major blood vessel involvement. J Vasc Surg 44(1):46-55

4. Kirchberg J, Reißfelder C, Weitz J et al (2013) Laparoscopic surgery of liver tumors. Langenbecks Arch Surg 398(7):931-938

5. Brenner T, Hofer S (2013) Point-of-care-testing and haemostaseological treatment algorithms. Anasthesiol Intensivmed Notfallmed Schmerzther 48(5):336-345

6. Bellemann N, Sommer CM, Mokry T et al (2014) Hepatic artery stent-grafts for the emergency treatment of acute bleeding. Eur J Radiol 83(10):1799-1803

7. Strobel O, Büchler MW (2015) Peritonealer Patch als autologer Venenersatz in der Pancreas- und hepatobiliären Chirurgie. Chirurg 86:1068

8. Hyhlik-Dürr A, Geisbüsch P, Hakimi M et al (2009) Endovascular aortic surgery: management of secondary aortobronchial and aorto-enteral fistulas. Chirurg 80:947-955

9. Shantikumar S, Patel S, Handa A et al (2011) The role of cell salvage autotransfusion in abdominal aortic aneurysm surgery. Eur J Vasc Endovasc Surg 42(5):577-584

10. Orendt KH (2012) Gefäßverletzungen. In: Debus ES, Gross-Fengels W (Hrsg) Operative und Interventionelle Gefäßmedizin. Springer, Berlin, S 825-838. (Kap. 40)

11. Kavic SM, Atweh N, Ivy ME et al (2001) Celiac axis ligation after gunshot wound to the abdomen.J Trauma 50:738-739

12. Goldstone J (1991) Anastomotic aneurysms. In: Bernard VM. Towne JB (Hrsg) Complications in Vascular Surgery. Quality Medical Publishing, St Louis

13. Dennis JW, Littooy FN, Greisler HP et al (1986) Anastomotic pseudoaneurysms: a continuing late complication of vascular reconstructive procedures. Arch Surg 121:314

14. Bastounis E, Georgopoulos S, Balas P (1996) The validity of current vascular imaging methods in the evaluation of aortic anastomotic aneurysms developing after abdominal aortic aneurysm repair. Ann Vasc Surg 10:537

15. Byrne DE, Crawford FA (1980) Traumatic vena cava injuries. Am J Surg 140:600-602

16. Carroll RC, Craft RM, Langdon RJ et al (2009) Early evaluation of acute traumatic coagulopathy by thrombelastography. Transl Res 154(1):34-39

17. Bandyk DF, Berni GA, Thiele BL et al (1984) Aortofemoral graft infection due to Staphylococcus epidermidis. Arch Surg 119:102
18. Back MR (2010) Local Complications-Graft Infection. In: Cronenwett JL (Hrsg) Ruherford's vascular surgery, 7. Aufl. Saunders Elsevier, Philadelphia

19. Bergqvist D, Bjorck M (2009) Secondary arterioenteric fistulation-a systematic literature analysis. Eur J Vasc Endovasc Surg 37:31-42

20. Armstrong PA, Back MR, Wilson JS et al (2005) Improved outcomes in the recent management of secondary aortoenteric fistula. J Vasc Surg 42:660666

21. Antoniou GA, Koutsias S, Antoniou SA et al (2009) Outcome after endovascular stent graft repair of aortoenteric fistula: a systematic review. JVasc Surg 49:782-789

22. Hertzer PJ, Beven EG, Krajewski LP (1986) Surgica management of infected abdominal aorticgrafts: review of a 25-year experience. J Vasc Surg 3:725731

23. Müller BT, Abbara S, Hennes N, Sandmann W (1999) [Diagnosis and therapy of second aortoenteric fistulas: results of 16 patients]. Chirurg 70:415-421

24. Mylona S, Ntai S, Pomoni M et al (2007) Aortoenteric fistula: $\mathrm{CT}$ findings. Abdom Imaging 32:393-397

25. Fiorani P, Speziale F, Rizzo L et al (1993) Detection of aortic graft infection with leukocytes labeled with technetium $99 \mathrm{~m}$-hexametazime. J Vasc Surg 17:87-95. (discussion 95-86)

26. Fukuchi K, Ishida Y, Higashi M et al (2005) Detection of aortic graft infection by fluorodeoxyglucose positron emission tomography: comparison with computed tomographic findings. J Vasc Surg 42:919-925

27. Hayes PD, Nasim A, London NJ et al (1999) In situ replacement of infected aortic grafts with rifampicin-bonded prostheses: the Leicester experience (1992 to 1998). J Vasc Surg 30:92-98

28. Kieffer E, Gomes D, Chiche L et al (2004) Allograft replacement for infrarenal aortic graft infection early and late results in 179 patients. J Vasc Surg 39:1009-1017

29. Linder F, Vollmar J (1965) The surgical treatment of acute arterial injuries and their sequealae. Hefte Unfallheilkd 81:38-53 